\title{
La inmigración en España: ¿Una integración con pies de barro?
}

\author{
Sara Moreno-Colom \\ Universitat Autónoma de Barcelona \\ (sara.moreno@uab.cat) \\ Ramon De Alós \\ Universitat Autónoma de Barcelona \\ (ramón.dealos@uab.cat)
}

Recibido: 09-02-2015

Aceptado: 10-11-2015

\begin{abstract}
Resumen
El objetivo del artículo es analizar el impacto de la crisis económica en la integración de la población inmigrada en España. El punto de partida es la singularidad del caso español que durante los años de intensa inmigración consiguió un notable grado de integración sociocultural. Se defiende que dicha integración ha sido el resultado de la confluencia de factores excepcionales alejados de una planificación y actuación política clara. A partir de una metodología mixta, se argumenta que durante el período de expansión, la relativa fácil inserción laboral de los inmigrantes y el acceso a los bienes y servicios públicos contribuyen a la convivencia con la población autóctona. Asimismo, se evidencia como la crisis económica, con sus efectos en términos de destrucción de empleo y políticas de austeridad, ponen al descubierto las debilidades del modelo español de integración.
\end{abstract}

Palabras clave: integración sociocultural; inserción laboral; ciudadanía social; convivencia; crisis económica; xenofobia.

\section{Immigration in Spain: Integration with Feet of Clay?}

\begin{abstract}
The aim of the paper is to analyze the impact of the economic crisis on the integration of the immigrant population in Spain. The Spanish case is singular because during the years of intense immigration achieved a remarkable degree of socio-cultural integration. The paper argues that such integration it has been the result of the confluence of exceptional factors rather than the result of the policy making. From a mixed methodology approach, it shows that, during the period of expansion, two factors of the immigration contribute to their coexistence with native population: finding job and access to public services. But the economic crisis, with its impact in terms of job losses and austerity policies, expose the weaknesses of the Spanish model of integration.
\end{abstract}

Key words: socio-cultural integration; employment; social citizenship; coexistence; economic crisis; xenophobia.

\section{Referencia normalizada}

Moreno Colom S. y R. De Alós (2016): "La inmigración en España: ¿Una integración con los pies de barro?", Política y Sociedad, 53 (2), pp. 509-528.

Sumario: Introducción. 1. La inmigración en España en el cambio de siglo. 2. El desencanto llega con la crisis. 3. Reflexiones finales. 4. Bibliografía. 


\section{Introducción}

El importante proceso de inmigración que recibe España desde finales del siglo pasado hasta entrada la crisis económica se ha caracterizado por un notorio nivel de integración de la población extranjera que contrasta con la experiencia de otros países dónde la alta inmigración se percibe como una amenaza (Portes y Rumbaut, 2010). Efectivamente, el caso español es peculiar por qué durante los años de intensa inmigración consiguió un notable grado de integración de dicha población a pesar de la falta de planificación y de una actuación política clara ${ }^{1}$ (Cebolla y González, 2013). Martínez de Lizarrondo (2009) describe el caso español como un modelo de integración patchwork dónde se dan variaciones de un mismo sistema que encajan en una política singular dentro de la UE: heterogeneidad autonómica dentro de un paraguas estatal; rasgos del régimen mediterráneo de bienestar; y diferenciación entre los sistemas migratorios de gestión temporal de la mano de obra e instalación sedentaria. En este sentido, distintos estudios evidencian que el modelo migratorio español está focalizado en las necesidades del mercado de trabajo y su gestión (Martín Patino, 2011). Este modelo vincula el mantenimiento de la situación regular de los inmigrantes a su cotización a la Seguridad Social y supedita el reconocimiento de derechos al permiso de trabajo.

Como han analizado Portes y Rumbaut (2010), los períodos de intensa inmigración suelen estar marcados por el temor entre la población nativa, de amenaza a la integridad de su cultura y de sus modos de vida. En España, dicho temor se podría identificar con el discurso de la invasión, aunque como apuntan Cebolla y González (2013), se trata de un discurso que no trasciende del imaginario de algunos colectivos y que no incide en las relaciones sociales bajo formas de conflicto y/o exclusión más allá de episodios puntuales de xenofobia. Entre las razones de este relativo éxito de integración destaca el ciclo de expansión económica con generación de empleo, el acceso a las prestaciones y servicios públicos y los procesos legales de regularización. La irrupción de la crisis económica supone un cambio de escenario que invierte la tendencia destruyendo empleo e introduciendo barreras administrativas y recortes en prestaciones y servicios públicos. Además de la falta de empleo y el debilitamiento de los derechos de ciudadanía social para muchos inmigrantes, también aumentan las tensiones y actitudes negativas ante la inmigración. Ante este escenario, el objetivo de este artículo es analizar el impacto de los primeros años de la crisis económica en la integración de la población inmigrada en España. Como objetivos específicos, se propone analizar la integración y el impacto de la crisis a partir de las trayectorias laborales, los discursos y las estrategias de las personas inmigrantes ante el contexto de destrucción de empleo, aumento del paro y mayor precariedad. En última instancia, se plantea hasta qué punto es posible la integración sin ocupación.

Para desarrollar el análisis, se parte del concepto integración sociocultural definido por Solé (1981) como un proceso por el cual los inmigrantes se insertan en la estructura ocupacional y aceptan progresivamente las instituciones, creencias, valores y símbolos

${ }^{1}$ La política de integración española se recoge en el I y el II Plan Estratégico de Ciudadanía e Integración (PECI) dónde se adaptan los principios del marco normativo de la Unión Europea con otras singularidades propias. 
de la sociedad receptora. Una propuesta teórica que la misma autora hace operativa en tres niveles de integración: estructural, con el acceso al empleo; cultural, con el ejercicio del derecho a la diferencia y aceptación de unos mínimos de convivencia; y jurídica, como garantía de igualdad ante la justicia (Solé et al., 2002). Esta propuesta es el punto de partida del artículo que adapta, a partir de la realidad del caso español, el concepto de integración sociocultural según tres dimensiones relacionadas de forma jerárquica: laboral, derechos de ciudadanía social y convivencia. Dada la importancia de la inmigración económica en España, se parte del nivel estructural que se equipara con la incorporación al mercado laboral de las personas inmigrantes. De modo que la cotización a la Seguridad Social, más allá del empadronamiento municipal, permite acceder a los derechos de ciudadanía social ${ }^{2}$, como prestaciones, pensiones y otras ayudas. En última instancia, se entiende que el hecho de tener trabajo y acceso a los derechos sociales conlleva un estatus civil que contribuye al "reconocimiento del otro" facilitando la convivencia con la población autóctona. A partir de esta propuesta teórica, el texto defiende que el contexto de crisis evidencia las debilidades del modelo de integración sociocultural construido, en buena parte, sobre la base del primer nivel de integración, es decir, la participación laboral de los inmigrantes. Por esta razón, se analiza el impacto de la crisis en las trayectorias laborales de los inmigrantes y, en segundo lugar, los cambios en sus estrategias y discursos migratorios.

Se propone una estrategia metodológica mixta. Por un lado, el grueso de la evidencia empírica sobre trayectorias laborales, estrategias y discursos proviene del análisis llevado a cabo a lo largo del año 2013 en el proyecto de investigación MIGRA ${ }^{3}$. El objetivo de este proyecto fue analizar las trayectorias laborales de la población inmigrante en los años de crisis económica a partir de una doble aproximación cuantitativa y cualitativa. La primera se centró en el análisis de la Muestra Continua de Vidas Laborales (MCVL) de la Seguridad Social (SS) de 2011; y la segunda en la realización de 14 entrevistas a líderes de organizaciones de inmigrantes, así como organizadores y gestores de políticas activas del mercado de trabajo y políticas sociales dirigidas a inmigrantes ${ }^{4}$. Por lo que se

${ }^{2}$ Es preciso diferenciar la ciudadanía social que supone el acceso a los bienes y los servicios públicos, de la ciudadanía política que representan los derechos y los deberes que conlleva el reconocimiento de sujeto político.

${ }^{3}$ Proyecto "Inmigrantes en la crisis económica ¿trayectorias laborales bloqueadas?", financiado por la Obra Social de "la Caixa". En la investigación participaron, además de los autores, Fausto Miguélez, Pedro López, Antonio Martín y Oscar Molina, todos ellos del Centre d'Estudis Sociològics sobre el Treball i la Vida Quotidiana (QUIT), de la Universitat Autònoma de Barcelona.

${ }^{4}$ A lo largo del año 2013, se entrevistó a representantes de 4 organizaciones de inmigrantes, 2 latinoamericanas (OI-1 y OI-2) y 2 africanas (OI-3 y OI-4); 3 responsables de la administración pública de Cataluña (AP-1, AP-2 y AP-3); 2 de organizaciones sindicales (OS-1 y OS-2); 3 de organizaciones empresariales (OE-1, OE-2 y OE-3); y de 2 consulados latinoamericanos en Barcelona (C-1 y C-2). Para más detalles, ver (autores, 2014). 
refiere a la $\mathrm{MCVL}^{5}$, ésta permitió una mirada retrospectiva de trayectorias laborales, de autóctonos e inmigrantes, teniendo en cuenta variables como sector de actividad, grupo de cotización, base de cotización, relación laboral, tipo de contrato, jornada, territorio, tiempo de permanencia en el mercado de trabajo, duración del contrato, además de sexo, edad, estudios y origen geográfico. El estudio se centró en la población de la MCVL del año 2011 que estaba de alta en la SS también en el año 2007, a efectos de observar su trayectoria laboral a lo largo de esos cuatro años. La aproximación cualitativa se diseñó para complementar la interpretación de los datos estadísticos y se centró en Cataluña porqué representa uno de los grandes territorios de atracción de inmigración en España (Miguélez y López-Roldán, 2014). Por el otro lado, el artículo también se ha servido de fuentes bibliográficas y estadísticas existentes ${ }^{6}$ tanto para completar el análisis de las trayectorias laborales como, sobre todo, las estrategias y discursos. Ello ha permitido introducir analíticamente indicadores objetivos acerca de las condiciones de vida de los inmigrantes e indicadores subjetivos en torno a los estados de opinión relativos a su integración.

El texto se estructura en dos apartados: el primero da cuenta de las características de la inmigración en España y el modelo de integración durante la fase de expansión económica. Sentadas estas bases, el segundo aparatado analiza el impacto de la crisis a partir de las trayectorias laborales, las estrategias y los discursos de la población inmigrante. Se concluye con unas reflexiones finales sobre el modelo de integración y sus retos de futuro.

\section{La inmigración en España en el cambio de siglo}

Numerosos estudios analizan el importante proceso de inmigración que ha conocido España sobre todo desde finales del siglo pasado y hasta entrada la gran crisis económica de este siglo (Cachón, 2009). Tres hechos suelen resaltarse. Primero, la gran afluencia de inmigrantes en esos años, de modo que "entre 1998 y 2008, el número de personas nacidas fuera de España pasó de representar el 2 al 16\%" (Cebolla y González, 2013: 36). Se trata de un aumento de la inmigración en España sin parangón entre los países de la Organización para la Cooperación y el Desarrollo Económico (OCDE) o de la Unión Europea (UE), según los mismos autores. Un segundo aspecto es que se trata, en su gran mayoría, de una inmigración económica, por motivos de búsqueda de empleo o mejora económica (Izquierdo, 2000; Solé, 2001; Cuadrado et al., 2007; Pérez Infante, 2009). Y como tercer aspecto se resalta el cambio en la procedencia en los flujos. En 1996 los extranjeros comunitarios suponían el $46 \%$ del total de extranjeros empadronados en

${ }^{5}$ Los microdatos de la MCVL se obtienen a partir de una muestra diseñada tomando como población de referencia a "todas las personas que han estado en situación de afiliado en alta, o recibiendo alguna pensión contributiva de la Seguridad Social en algún momento del año de referencia" (Ministerio de Trabajo e Inmigración, 2009: 3).

${ }^{6}$ Básicamente la Encuesta de Población Activa (EPA) y la Encuesta Nacional de Inmigrantes 2007 (ENI), ambas del Instituto Nacional de Estadística (INE), y las encuestas sobre Actitudes hacia la inmigración del Centro de Investigaciones Sociológicas (CIS). 
España, mientras que en 2003 ya sólo suponen el 22\% (Consejo Económico y Social, 2004). Entre los no comunitarios, desde los años 90 una parte destacada de los mismos procedía de África, en su gran mayoría marroquíes (Cachón, 2009), posteriormente, a partir del año 2000 , se añade un fuerte contingente originario desde diversos países latinoamericanos, con un importante componente femenino (Cebolla y González, 2013). La población procedente de Rumanía experimenta un fuerte aumento a partir de 2004, de modo que en pocos años constituye la segunda comunidad de inmigrantes en número de residentes en España (750.901) según el censo de población de 2011, tras los nacidos en Marruecos (772.126).

Varias explicaciones concurren en dar respuesta a los importantes flujos migratorios que ha conocido España en el cambio de siglo. Sin olvidar la importancia que puedan tener la situación económica y del empleo, también la demográfica, de los países emisores, así como en su caso las crisis políticas, la mayoría de estudios referidos a España se centran en tres aspectos de atracción: el crecimiento económico y aumento de la demanda de empleo, los cambios en cuanto aceptabilidad de un empleo por parte de la población autóctona y la legislación.

Algunos autores resaltan el fuerte aumento del PIB y del empleo (Pérez Infante, 2009), bastante superior a la media de la UE-25, y ante una reducción de la tasa de natalidad (Pajares, 2007; Pérez Infante, 2008: 130; OECD, 2009). Otros se remiten al efecto atracción inducido por el desajuste que se produce en el mercado de trabajo español desde mediados de los años 80 como consecuencia del aumento del nivel de aceptación de las condiciones de empleo por parte de los jóvenes españoles (Cachón, 2009). La mejora de las condiciones laborales de los autóctonos explicaría, pues, la fuerte entrada de inmigrantes en determinadas actividades (Cachón y Valles, 2003). De ahí que la población inmigrante, con un nivel de aceptación de empleos más laxo, se concentre en aquellas actividades menos deseables para los autóctonos (Cachón, 2012; Diaz-Serrano, 2013).

Por su parte, Cebolla y González (2013) llegan a la conclusión que siendo el crecimiento económico una causa determinante de la inmigración, no es suficiente. Los autores defienden que la exención del visado a los nacionales de muchos países latinoamericanos explica el por qué la inmigración latinoamericana haya aumentado de forma tan espectacular, especialmente entre 2000 y 2005. Coincidiendo con "el endurecimiento de la política de inmigración en otros países receptores como Estados Unidos, pero también la defectuosa regulación y gestión que se hizo en España del hecho migratorio durante la mayor parte del periodo analizado" (2013: 61). En definitiva, si Izquierdo (1996) calificó de inesperada la inmigración que llegó a España hasta primeros de los 90, hoy puede afirmarse que gran parte de la inmigración posterior a esa fecha ha sido esperada.

\subsection{La inmigración esperada en el mercado de trabajo}

El importante flujo de población inmigrada por motivos económicos en apenas dos décadas justifica analizar el nivel de integración alcanzado en España considerando la dimensión laboral como factor explicativo clave. En este sentido, destacamos tres 
aspectos: la relativamente fácil ${ }^{7}$ inserción laboral de los inmigrantes, la no competencia de empleos con los nativos y el haber facilitado el acceso al empleo de mujeres autóctonas.

En primer lugar, cabe afirmar que una buena parte de la población inmigrante ha tenido un relativamente fácil acceso al empleo, independientemente de las características de estos empleos en términos de estabilidad, salario, salud laboral, seguridad, cobertura social, cobertura de la negociación colectiva y de representación sindical, y sin descartar los episodios, más o menos frecuentes, de desempleo. Pajares (2007), en base a la EPA del último trimestre de 2006, muestra como la tasa de actividad de la población extranjera en España es muy elevada, 77,6\% frente a 58,6\% para el conjunto de la población; asimismo lo es la tasa de empleo, $68,3 \%$ y 53,7\% respectivamente, aunque también lo es la tasa de paro, $12,0 \%$ y $8,3 \%$ respectivamente. Esta diferencia en la tasa de paro pone en evidencia unas mayores dificultades de inserción laboral. Según INE (2009) hasta el 53,8\% de los nacidos en el extranjero que residía en España en el 2007 ha tenido alguna experiencia de paro desde su llegada, lo que es indicador de su elevada inestabilidad laboral. Con todo, merece destacarse una tasa de empleo entre la población extranjera a fines del año 2006 cerca de 15 puntos superior a la media nacional. Ello significa que la población inmigrante mantiene una relación mucho más intensa con el mercado laboral que la autóctona. Pero no sólo hay que destacar el acceso al empleo; también el horizonte de su mejora abierto para muchos inmigrantes. Son diversos los estudios que relacionan las perspectivas de movilidad ascendente con el tiempo de residencia en España (Fernández y Ortega, 2008; Pérez y Serrano, 2008; INE, 2009; Diaz-Serrano, 2013). Concretamente, esta relación se evidencia con el análisis de la MCVL centrado en los itinerarios que siguen los individuos (autóctonos e inmigrantes) desde su entrada en el mercado de trabajo hasta diciembre de 2007 (Miguélez, et al., 2011). En dicha investigación, anterior a la que sirve de base empírica para este artículo, se concluye que una mayoría de los inmigrantes mejora su situación, aunque algunos lo hacen más que otros, siendo la razón de estas diferencias no la procedencia sino atributos de tipo individual como el tiempo que llevan en el mercado laboral español, la lengua y el nivel de estudios. Se trataría de una movilidad segmentada, según AysaLastra y Cachón (2013) analizando los datos de la ENI, pues se daría dentro de cada segmento del mercado laboral. Con todo, las probabilidades de movilidad ascendente de la población inmigrada no deben desfigurar una realidad marcada por la discriminación en un mercado de trabajo segmentado y segregado. Es preciso señalar su inserción en nichos laborales propios del segmento secundario del mercado laboral, en empleos comparativamente más precarios, inestables, peor remunerados, más vulnerables y más expuestos a las arbitrariedades de mandos y empresarios, en algunos casos dentro del ámbito de la economía sumergida (Miguélez et al., 2011). Una segmentación que se sobrepone a una segregación en función del sexo (Solé, 2001).

${ }^{7}$ Se utiliza el adjetivo fácil para referirse al hecho de que la inserción laboral de los inmigrantes se da en un periodo relativamente corto de tiempo desde la llegada en el país receptor sin dificultades atribuibles a procesos exclusivamente discriminatorios. 
El segundo aspecto a resaltar es que, como destacan diversos autores, la población autóctona, en general, no se ha visto forzada a competir por unos mismos puestos de trabajo que los inmigrantes, puesto que unos y otros difieren en sus ubicaciones en la estructura de sectores de actividad y de ocupaciones laborales (Cuadrado et al., 2007). Carrasco y Ortega (2005) y Pajares (2007) descartan un panorama general de competencia entre autóctonos e inmigrados en el que los primeros pierdan oportunidades de empleo por la llegada de los segundos. Cebolla y González constatan que la llegada de inmigrantes durante el período 2001-2006 no se relaciona con el desplazamiento de autóctonos hacia el desempleo, ni con una caída de los salarios (2013). Es más, Pajares apunta a una situación en la que inmigrantes y autóctonos no competirían por unos mismos puestos de trabajo, sino que los segundos encontrarían nuevas oportunidades de empleo en categorías intermedias y altas, gracias a un desarrollo económico que es posible porque los inmigrantes acceden a las categorías bajas (2007-2008).

Como tercer aspecto, destaca que la llegada de mujeres inmigrantes ha permitido un mayor acceso al empleo de mujeres autóctonas. Los bajos salarios y la elevada disponibilidad horaria han permitido que numerosas familias autóctonas pudieran acceder a contratar, más o menos formalmente, a mujeres inmigrantes en tareas de servicio doméstico y/o de atención a personas mayores. Cebolla y González destacan que el abaratamiento del servicio doméstico "hizo que en las regiones de más inmigración, aumentara también el empleo de mujeres autóctonas más cualificadas, es decir, con titulación universitaria y responsabilidades familiares" (2013: 110). Parella (2003) argumenta que la feminización de los flujos migratorios procedentes de países latinoamericanos está íntimamente relacionada con los factores de atracción, esto es, la demanda de trabajadoras inmigrantes para insertarse en el servicio doméstico. Esta externalización del trabajo reproductivo resulta una práctica muy extendida en los países del sur de Europa dadas las debilidades de sus regímenes de bienestar y su carácter familista (Sarasa y Moreno, 1995).

\subsection{La integración inesperada: derechos y convivencia}

Como se explica en la introducción, este artículo defiende que el acceso al empleo es condición necesaria para garantizar los derechos de ciudadanía de la población inmigrante y la convivencia con la población autóctona. Por un lado, durante el período de expansión, la relativa fácil inserción de los inmigrantes en el mercado laboral facilitó su acceso a los servicios de sanidad, educación y vivienda, además de otros servicios y prestaciones sociales. Así, por ejemplo, merece citar la integración de los menores en el sistema educativo español, y en particular de los recién llegados y la acogida de hijos de inmigrantes no regularizados; una cuestión no menor si se tiene en cuenta que, según el Censo de Población de 2011, el 15\% de la población de nacionalidad extranjera no llegaba a los 16 años de edad. No obstante, no debe olvidarse que los hijos de los inmigrantes en España obtienen peores resultados en rendimiento escolar (Cebolla y González, 2013); problemas derivados de su concentración en determinados barrios y centros escolares (Pajares, 2005; Moreno y Bruquetas, 2011), y agravado por la carencia de objetivos a medio y largo plazo en política educativa (Cebolla, 2010). 
También cabe resaltar las posibilidades de acceso de la población inmigrante al sistema sanitario, tras su universalización. Aunque algunos autores destacan la existencia de dificultades motivadas por desconocimiento, idioma, pautas culturales, o para la población inmigrante indocumentada (Moreno y Bruquetas, 2011).

Por lo que se refiere a la vivienda, la fuerte llegada de inmigración en España ha coincidido en el tiempo con el boom inmobiliario. Si tras la llegada al país de acogida, alquilar una habitación puede haber sido una opción generalizada (Leal y Alguacil, 2012), estos mismos autores destacan como a medida que las condiciones de vida de los inmigrantes han mejorado, también lo ha hecho su condición residencial. Ello no impide que, sobre todo en las grandes ciudades, se haya producido una concentración de población inmigrante en los barrios más degradados o marginales, sin que ello, por lo general, haya conllevado la formación de guetos o zonas espacialmente diferenciadas de la población nativa: apenas un $20 \%$ de los inmigrantes vive en comunidades en las que más de la mitad de los vecinos también lo son (Colectivo Ioé, 2006). No se dan, pues, niveles de segregación residencial tan altos como en otros países europeos (Cebolla y González, 2013).

Finalmente, cabe mencionar que aunque el gobierno español ha carecido de políticas para promover una plena integración de ciudadanía social de los inmigrantes hasta la aprobación de la Ley Orgánica 2/2009 sobre derechos y libertades de los extranjeros en España y su integración social (Cebolla y González, 2013), desde comunidades autónomas, municipios y otras instituciones se han desarrollado planes que han contribuido a su integración. A pesar de la falta de una estrategia clara y coherente de lo que Martínez de Lizarronda (2009) identifica como el modelo patchwork de integración, destacan proyectos en el nivel autonómico y local como: intervenciones de acogida, de ayuda al acceso a la vivienda, de apoyo a la inserción laboral, de atención a la mujer inmigrada, de salud y acceso a la sanidad, de atención a los menores no acompañados, de educación y escolarización de los menores inmigrados, de formación de mediadores (Pajares 2005). Aunque también aparecen algunas limitaciones que permiten suponer un menor acceso de la población inmigrante a determinados servicios, como, por ejemplo, la renta mínima de inserción (Moreno y Bruquetas, 2011).

Por el otro lado, las características de la integración laboral de la población inmigrante y su nivel de integración ciudadana han contribuido al tercer nivel de integración, la de convivencia, que complementa las tres dimensiones de la integración sociocultural. La limitada competencia por unos mismos puestos de trabajo entre trabajadores autóctonos e inmigrantes, también por unos mismos servicios y prestaciones, y el hecho de que la inmigración femenina haya facilitado el acceso al mercado laboral de mujeres autóctonas puede sugerir unos niveles elevados de aceptación de la población inmigrante por parte de la autóctona. Posiblemente más en el ámbito laboral que en el residencial, en la medida en que en el primero la complementariedad ha sido mayor que en el segundo ámbito (Jódar et al., 2014). De hecho, salvo algunos episodios esporádicos de xenofobia, los niveles de convivencia entre población inmigrante y autóctona en España han sido relativamente elevados (Cebolla y González, 2013). Como expone Subirós: "la misma sociedad que recibe a los inmigrantes con temores y recelos, con estereotipos, es la misma que los necesita, que sin ellos no podría funcionar "(2010:38). 
Por su parte, Morales et al. (2008), en su estudio centrado en Barcelona y Madrid, hallan actitudes generalmente positivas por parte de los diversos colectivos de inmigrantes hacia los españoles y hacia las instituciones, al mismo tiempo que unos niveles de confianza social relativamente similares a los de la población española autóctona. No obstante, no puede olvidarse que la visibilidad de los rasgos étnicos interfiere en los procesos de incorporación de los inmigrantes en la sociedad de acogida (Cea D'Ancona et al., 2014). Así lo constatan Morales et al. (2008) en las mayores dificultades del colectivo marroquí para interactuar con la población autóctona y su percepción de discriminación por razón de procedencia, color de piel o religión.

Esta integración sociocultural desde la perspectiva de las personas inmigradas se refuerza en la medida en que entre las mismas se genera un estado de illusio, en términos de Bourdieu (1994). Es decir, la creencia de que ha tenido sentido su proyecto y decisión migratoria, de que ha valido y merece la pena el cambio realizado en sus vidas, pese a sus innegables dificultades personales, familiares y económicas. Así se deduce del estudio del INE (2009) que pone de manifiesto como una buena parte de los inmigrantes ha conseguido mejorar sus condiciones de empleo y de vida con relación a las condiciones en su país de origen.

Diversos estudios cualitativos apuntan que una buena parte de la inmigración llegó sin un proyecto migratorio concreto, con la expectativa de mejorar o de huir de situaciones extremadamente difíciles en sus países de origen (Subirós, 2010). Frases como "el boliviano no vino para quedarse", expresada por una persona de OI1 , dan cuenta de un proyecto migratorio transitorio que se concretará con el paso del tiempo. De ahí que ya en 2007, el 80,6\% de los inmigrantes expresara el deseo de permanecer en España, frente a un 6,7\% que pensaba volver a su país de nacimiento en los próximos cinco años (INE, 2009). El mismo estudio del INE añade que hasta un 24,8\% de los entrevistados (año 2006) tenía la intención de traer familiares a España. En este sentido, las estadísticas muestran un importante aumento de los procesos de reagrupación familiar a lo largo de los primeros años del siglo XXI. Así, las solicitudes que cumplían con los requisitos legales han evolucionado de 7.197 en el año 2000 a 144.584 en el 2007 (González Ferrer, 2009), lo cual es indicativo del asentamiento de la población inmigrada en el país de acogida. El conjunto de elementos descritos explica cómo se ha contribuido a una "integración inesperada" (Cebolla y González, 2013:162).

\section{El desencanto llega con la crisis}

En la medida que la integración sociocultural se ha sustentado, en buena parte, en las oportunidades de acceso a un empleo de los inmigrantes, resulta necesario analizar el impacto que supone la irrupción de la crisis económica sobre la misma. En otras palabras, surge la necesidad de saber ¿qué ocurre con la integración cuando se debilita el nivel estructural que representa el empleo? La situación de paro coloca en la cuerda floja a los inmigrantes para acceder a las prestaciones sociales y renovar su permiso de trabajo, condiciones que apuntan a los peligros de la irregularidad y la xenofobia. Con el fin de avanzar en dicha cuestión, se analiza el impacto de la crisis a partir de 
las trayectorias laborales, las estrategias y los discursos de los inmigrantes fijando la atención en el mercado de trabajo, los derechos sociales y la convivencia.

\subsection{Trayectorias laborales bloqueadas}

Son conocidos los efectos devastadores que tiene la crisis en el mercado de trabajo especialmente entre la población inmigrante. De acuerdo con la EPA, a partir del tercer trimestre de 2007 y hasta finales de 2013 se han perdido 3.618.200 empleos, de los cuales uno de cada cuatro corresponde a inmigrantes. Y la tasa de paro se ha elevado hasta el $25,7 \%$, el 36,6\% para la población extranjera. Por otra parte, el porcentaje de hogares de inmigrantes con todos sus miembros activos en paro ha pasado del 3,9\% en 2007 al 15,3\% en 2011, alcanzando al 29,1\% de los hogares de africanos ese último año (Colectivo Ioé, 2012).

Sin embargo, como advierten diversos estudios, la crisis no afecta a todas las personas inmigrantes por igual. La segmentación y la segregación del mercado de trabajo, no siempre visible en la época de expansión, hace mella con la crisis. Se refuerza la segregación ocupacional y la especialización por género con la crisis, aumentando la concentración de la población inmigrante en empleos de baja cualificación y mayor especialización en actividades y ocupaciones concretas (Pajares, 2010; Garrido et al., 2010; Muñoz, 2011; Staneck, 2011; Carrasco y García, 2012).

El análisis realizado en el proyecto MIGRA con los datos de la MCVL acumulados hasta 2011 refuerza los resultados indicados y apunta otros adicionales, sobre todo en términos de trayectorias laborales. A grandes rasgos, puede decirse que el 27,7\% de los inmigrantes con empleo en el año 2007 están en paro cuatro años después, en 2011, y el $46,1 \%$ de los que estaban en paro entonces también lo están en 2011. Durante el primer periodo de crisis, esta conlleva un retroceso en las trayectorias laborales de los hombres inmigrantes, sobre todo, de los originarios de Ecuador y Marruecos; la principal razón recae en la destrucción de empleo en sectores como la construcción, que en los años anteriores a la crisis ocupó porcentajes elevados de hombres inmigrantes procedentes de ambos países: aproximadamente uno de cada dos inmigrantes empleados en la construcción a finales del 2007 se hallaba sin empleo a finales del 2011. Se observa también un bloqueo en las trayectorias laborales de las mujeres inmigrantes mayores de 45 años y con poca formación. Y los cambios territoriales y sectoriales más significativos entre la población inmigrante se deben leer en clave de supervivencia. Así, por ejemplo, la mayor presencia de hombres inmigrantes marroquíes en el régimen agrario de la SS, donde habrían encontrado empleo al ser expulsados como trabajadores del régimen general y del régimen de autónomos en el sector de la construcción. Por su parte, las mujeres latinoamericanas y marroquíes se mantienen como las principales trabajadoras dentro del régimen de empleadas del hogar; las primeras principalmente empleadas como cuidadoras de personas dependientes y las segundas como limpiadoras del hogar (Miguélez y López-Roldán, 2014).

Si entre 2007 y 2011 un 34,7\% de los trabajadores autóctonos ha experimentado un periodo de desempleo y/o sin relación con la SS superior al año, el porcentaje para los inmigrantes asciende al 51,6\%. Dentro de estos últimos, un 32,8\% ha tenido periodos de muy larga duración de carencia de empleo, superior a dos años. La fuerte intensidad 
y larga duración de la crisis económica ha producido un aumento significativo del desempleo y, en particular, del desempleo de larga duración. La MCVL sólo permite captar parcialmente este fenómeno debido a que únicamente figuran como desempleados los trabajadores que perciben prestación contributiva o subsidio por desempleo. No obstante, agregando el tiempo de desempleo y el tiempo sin relación con la SS de cada individuo, como aproximación a la extensión y características del posible desempleo de larga duración, los resultados apuntan que haber experimentado un periodo de carencia prolongada de empleo se traduce en situaciones de bloqueo y en muchos casos en trayectorias laborales descendentes.

Los datos de la MCVL ponen de manifiesto la influencia del género en la actual coyuntura económica, aunque actúa en sentido contrario al habitual. Puesto que la mayor destrucción de empleo se ha dado en los sectores tradicionalmente masculinizados, los hombres experimentan una mayor pérdida de empleo en sus trayectorias laborales en comparación a las mujeres. Por el contrario, las mujeres resisten mejor el impacto de la crisis gracias, paradójicamente, a la segregación horizontal que las emplea mayoritariamente en el sector servicios, especialmente en los que se refieren a la atención a las personas. Pero la segregación horizontal emerge como la doble cara de la moneda del empleo femenino, porque también supone que los empleos que ocupan las mujeres duran menos tiempo y son más precarios. En definitiva, las mujeres resisten mejor el contexto de recesión, aunque sus trayectorias aparecen bloqueadas por las desigualdades de género persistentes en el mercado de trabajo y las desventajas que acompañan la estrategia migratoria. La MCVL también apunta que algunas mujeres se habrían visto obligadas a trabajar más horas para compensar la pérdida salarial de sus parejas, ejerciendo el rol de principales mantenedoras económicas del núcleo familiar; un incremento de horas que aumentaría sus ingresos. No obstante, cabe precisar que la disminución en las diferencias de la base salarial según género debe atribuirse sobre todo al empeoramiento de las condiciones laborales de los hombres y no a la mejora de las condiciones laborales de las mujeres.

Así pues, parece posible afirmar que en comparación a 2007, último año del periodo de expansión económica, el empleo de los inmigrantes durante el primer período de la crisis económica (hasta 2011) es más femenino y más precario. La crisis acorta, a la baja, las diferencias de género relativas a la situación laboral: se equiparan las cifras de paro, se generaliza la temporalidad y disminuye la brecha salarial. Pero esta feminización precaria del empleo no impide que éste continúe segregado por razón de género. Así lo muestra el análisis de las variables relativas al tipo de jornada, sector de actividad y categoría laboral.

\section{2. ¿Integración sociocultural en tiempos de crisis?}

El fin de la integración laboral de los inmigrantes, con el aumento del paro, especialmente de larga duración, y la precariedad en el mercado de trabajo, plantean dudas sobre la integración sociocultural de los mismos: ¿qué pasa con los derechos de ciudadanía de los inmigrantes y la convivencia con la población autóctona cuando desaparece el empleo? A continuación, se analizan las estrategias y los discursos de los inmigrantes ante la crisis a partir de las entrevistas realizadas y otras fuentes secundarias. Tres 
aspectos apuntan las debilidades de un modelo de integración algo sobrevenido durante la expansión económica: cambios en las condiciones de vida y las estrategias migratorias como indicador de la pérdida de derechos de ciudadanía; y leve aumento en las posiciones críticas de la población autóctona sin afectar, aparentemente, a la convivencia.

\subsubsection{De las condiciones de vida a las estrategias migratorias}

Distintos estudios evidencian como la población inmigrante percibe los efectos adversos de la crisis (Carrasco y García-Serrano, 2015). Aunque en sus inicios la crisis afectó menos a los inmigrantes por su mayor disponibilidad a desplazarse (S$2)^{8}$, el parón sobre todo en la construcción expulsó a muchos de ellos, sobre todo a hombres, al desempleo (AP-2, AP-3, S-2, C-2). Y para los que mantienen un empleo se destaca un empeoramiento de sus condiciones, en salarios, alargamiento de la jornada, hasta en situaciones de impago del salario (C-1, OI-3). Diversos entrevistados concuerdan en que "el inmigrante lo está pasando mal" (OI-1, OI-3). Las encuestas del CIS a población inmigrante en 2011 confirman que el 86,3\% de éstos en Barcelona y el $82,4 \%$ en Madrid manifiestan tener problemas de ingresos para vivir, y de ellos, $5,9 \%$ y el $9,4 \%$, respectivamente, expresan muchas dificultades. Son aspectos también constatados por Colectivo Ioé (2012): si en el año 2007 el 41,1\% de los hogares con persona principal extranjera extra comunitaria tenía dificultades para llegar a final de mes, en el año 2011 asciende al 53,9\%. Cuando no se dispone de ingresos o de ingresos suficientes se multiplican los problemas, de compra de comida, de ropa, de pago de alquileres o pago de hipoteca (OI-1, OI-3, S-1). Cáritas confirma que la pobreza se desarrolla especialmente entre la población inmigrante: casi la mitad de las personas atendidas por la entidad en 2012 eran inmigrantes (2013). A ello se añade que si bien la crisis acrecienta las necesidades de protección y servicios públicos, se han aplicado importantes recortes en las ayudas a las organizaciones orientadas a la ayuda social y a la integración sociocultural "cuando más lo necesitan" (OI-1, OI-2, OI-4 y S-2). Como afirman Alguacil (2012) y Antón (2013) la gestión política dominante, de carácter neoliberal, ha adoptado medidas de ajuste y austeridad, con limitación del gasto público social y de los niveles de protección social, en particular de los servicios públicos, comprometiendo la universalidad de los derechos y la igualdad de acceso a los servicios.

Para una buena parte de la población inmigrante se desvanecen, pues, los procesos y perspectivas de integración laboral, que para muchos de ellos constituyó la principal motivación de su decisión de emigrar (Viruela, 2013). Aspecto en el que coinciden prácticamente todos nuestros entrevistados. Además, como expone Colectivo Ioé (2013), la crisis del empleo está suponiendo un aumento de "irregulares sobrevenidos". Pero no sólo son problemas económicos: a ello se añaden las restricciones añadidas en los trámites administrativos para conseguir la reagrupación familiar (S-1) o las dificultades de regularización a través del arraigo en tiempos de crisis (Domingo et al., 2012).

\footnotetext{
${ }^{8}$ Ver nota 3 para la identificación de las personas entrevistadas.
} 
Según OI-2, para muchos inmigrantes "la crisis ha roto el proyecto migratorio", mientras "para los familiares reagrupados domina más la sensación de que todo ha salido mal" (OI-4). Como concuerdan estos entrevistados, todo ello supone un golpe emocional muy fuerte, sobre todo para quienes llevan más años en España: "cuando un inmigrante está en paro, todos los familiares lo sufren, también los hijos" (OI-4). Dificultades que se acrecientan cuando se añade "el problema del desahucio" (OI2). Las diversas entidades entrevistadas concuerdan en que ha aumentado mucho su trabajo de atención a personas inmigrantes, en un contexto, como se ha expuesto, de reducciones drásticas de recursos financieros; "el trabajo de acogida, pues, resulta mucho más difícil cuando más se necesita” (OI-2).

Las entrevistas realizadas en el proyecto MIGRA apuntan cuatro estrategias principales de los trabajadores inmigrantes para hacer frente a las situaciones de desempleo y carencia de ingresos. La primera, una movilidad sectorial y geográfica; la agricultura y en menor medida la restauración, hostelería y el comercio habrían desempeñado un papel de flotador para muchos trabajadores. La segunda estrategia, en la que coinciden prácticamente todos los entrevistados, es el recurso al empleo informal "como instrumento de supervivencia para personas que se encuentran en situación de desempleo" (OE-1); sin embargo, algún entrevistado (OI-4, AP-1 y S-1) añade que aspectos administrativos relacionados con la obtención de permisos de residencia o de un contrato de trabajo (S-2), o incluso la obtención de la doble nacionalidad (C1), impulsaría a muchos inmigrantes a aceptar un empeoramiento sustancial en sus condiciones de empleo si esto les permite seguir vinculados formalmente a la Seguridad Social, antes que involucrarse en la economía sumergida. Como expone el entrevistado de C-1, con sueldos de 600 o 700 euros y exigiendo más horas de trabajo "ya no se puede hablar de mileurista". Una tercera estrategia es el recurso a las redes sociales, familia, amigos, comunidad y organizaciones de origen (OI-3 y OI-4); una solidaridad que tiene mucho peso en determinadas culturas (OI-2) y que ha aumentado con la crisis (OI-3). Finalmente, una cuarta estrategia consiste en la re-emigración o el retorno; aunque obviamente para las personas entrevistadas la cuantificación del fenómeno sea difícil, sí que se extraen algunas conclusiones. En primer lugar, el retorno ha sido una última opción para una parte de la población inmigrante; el retorno puede identificarse como un fracaso personal, pues "lo que esperan del que emigra es el éxito" (OI-4) o "volver con las manos vacías es un fracaso" (OI-3). En segundo lugar, la re-emigración, por ejemplo a Europa, se perfila como una estrategia de los grupos más jóvenes, debido a su mayor cualificación y a las expectativas que trabajar en Europa les puede abrir de cara al desarrollo de su carrera profesional (AP-2 y OI-4). Varios entrevistados coinciden en que la re-emigración o el retorno comportan la separación de familias. En palabras del representante de C-2, "después de una década, se han ido consolidando procesos de reagrupación familiar en España; pero, a partir de la crisis, vuelve a romperse la unidad familiar, pues en muchos casos la familia no tiene los recursos suficientes para retornar a todos sus miembros". Para C-2, OI-2, OI-4 y S-1 las familias ya reagrupadas suelen resistirse más a retornar por el impacto escolar y emocional que el abandono del país puede tener para los menores; aspecto destacado también por Cebolla y González (2013). Si la emigración latinoamericana tenía "rostro de mujer... ahora es el marido 
el que se tiene que mover o retornar" (C-1), pues es el que ha perdido el empleo. La vuelta al país de origen suele plantearse como el fin de una etapa migratoria: "de los que se han ido (a Bolivia) ninguno tiene la intensión de regresar" (OI-1). Así lo confirman Parella y Petroff (2014) cuando constatan que desde el año 2007 aumentan exponencialmente las salidas de extranjeros de España pero, añaden las autoras, un $11 \%$ de las bajas registradas en 2012 no superaban los 15 años de edad, lo que indica una estrategia familiar no temporal.

\subsubsection{El discurso de la competencia en la convivencia}

Por lo que se refiere a la convivencia, el crecimiento del desempleo aumenta la competencia con la población autóctona por unos mismos puestos de trabajo: existe al menos el sentimiento de que los españoles desplazan a los extranjeros, que pasan al paro (C-1). Asimismo, crece la competencia por unos recursos sociales más escasos (OI-1). Como consecuencia de todo ello se observan cambios por lo que respecta a las actitudes de autóctonos con respecto a la población inmigrante en España. Distintos estudios constatan que la crisis supone un aumento de las reticencias de la población española con relación a la posición de los inmigrantes en el mercado de trabajo y su acceso a las prestaciones y servicios públicos (Méndez et al., 2013). Algunos indicadores al respecto se recogen en las encuestas del CIS sobre "Actitudes hacia la inmigración". La comparación de sus versiones correspondientes a los años 2008 y 2012 permite constatar que quienes están muy de acuerdo con la opinión de que los inmigrantes quitan puestos de trabajo a españoles asciende del $19,9 \%$ al $28,3 \%$; o que bajan los salarios pasa del 30,1\% al 35,8\%. Por ello aumenta también (del 14,4\% al $17,7 \%$ ) quienes están muy de acuerdo con que si se quedan en el paro mucho tiempo deberían ser expulsados del país. Quienes opinan que no deberían tener derecho a traer a su familia pasan del 13,3\% al 15,6\%. Más en general, si quienes tenían una visión positiva de la inmigración en 2008 era el 45,8\% de la muestra, en 2012 es el 39,6\%. Resultados similares se constatan por lo que se refiere a la convivencia vecinal y la utilización de servicios sanitarios, educativos y sociales. Estos ascensos en el rechazo de la inmigración que registran los barómetros del CIS coinciden, como evidencian distintos estudios, con el protagonismo que adquiere la inmigración como problema en el ámbito de la política y en los medios de comunicación (Garcés et al. 2012; Cea D’Ancona y Valles, 2011; Cebolla y González, 2013).

Por otra parte, aumenta entre inmigrantes el sentimiento de rechazo en el país de acogida. Como explican las personas entrevistadas de OI-4, al inmigrante ahora se le ve más en las plazas, parques, servicios de urgencia, en las oficinas de empleo,...; en definitiva, es más visible, lo que "genera problemas". En este sentido, la crisis crea las condiciones idóneas para que algunos dejen de lado la corrección política y se sientan legitimados para expresar abiertamente el discurso de la preferencia "primero se ayuda a los de casa y después a los de fuera" (Cea d'Ancona et al., 2014: 30).Aumentan, pues, las actitudes contrarias o negativas hacia la inmigración; aunque por los datos del CIS mostrados cabe decir que muchas de ellas siguen siendo minoritarias. Así es confirmado por Cea D'Ancona et al. (2014), para quienes sigue predominando un racismo de baja intensidad en una realidad yuxtapuesta dónde se dan argumentos xenófobos y xenófilos. 
El contexto de crisis económica conlleva un leve aumento de discursos reacios y actitudes críticas hacia la inmigración al tiempo que se registran episodios positivos de convivencia visibles en los contactos cotidianos. Como concluyen Méndez et al.: "los españoles muestran actitudes ante la inmigración más positivas que las de otros países con nueva inmigración con dificultades económicas, como Portugal o Grecia" (2013).

\section{Reflexiones finales}

Los intensos flujos migratorios de los últimos años en España se han caracterizado por un notorio nivel de integración de la población extranjera que contrasta con la experiencia de otros países dónde la alta inmigración se ha percibido como una amenaza (Portes y Rumbaut, 2010). En el caso español, el contexto de bonanza económica con crecimiento y generación de empleo es un aspecto clave para entender cómo el inesperado volumen de inmigración (Izquierdo, 1996) se convierte en una inmigración esperada desde la perspectiva del mercado de trabajo. Básicamente porque permite cubrir los empleos menos valorados en los sectores de la construcción y los servicios, al tiempo que facilita la presencia laboral de mujeres autóctonas con la externalización de una parte del trabajo doméstico y de cuidados a través de la mano de obra inmigrante femenina. A pesar de no contar con un modelo definido de integración, la relativamente fácil inserción laboral de las personas extranjeras asegura el primer nivel de integración que condiciona positivamente la posterior convivencia con la población de acogida. En buena parte, porque la illusio de las personas recién llegadas en el acierto de su proyecto migratorio, a pesar de los costes personales, encaja con las necesidades de las personas autóctonas en términos de trayectorias laborales, personales y también familiares. Dicho encaje yace en el trasfondo de una integración sin modelo político claro dónde el empleo de unos y otros los equipara en relación a los derechos sociales de ciudadanía garantizando un clima de convivencia notable. Pero la misma clave del éxito de la integración en España puede convertirse en la razón de su fracaso en el contexto de crisis económica: ¿es posible la integración sin ocupación?

A partir del 2008, la irrupción de la crisis conlleva destrucción de empleo y precarización del mercado laboral debilitándose la principal vía de integración de la inmigración. Lo que se consideraba una relativa fácil inserción laboral durante el período de expansión, emerge como una relación débil con el mercado de trabajo marcada por la segmentación y la segregación. Las trayectorias laborales de las personas inmigrantes, especialmente de los hombres, son los más perjudicadas por el impacto de la crisis. La pérdida de empleo dificulta el acceso a las prestaciones y la renovación del permiso de trabajo. Junto estas trabas administrativas, crece la vulnerabilidad de estos colectivos que ven cada vez más difícil las estrategias de reinserción laboral al entrar en competencia con la población autóctona. Todo ello tiene lugar en un escenario político marcado por las políticas de austeridad y los recortes en las prestaciones y los servicios públicos. Se desvanece el encaje entre la illusio en los proyectos migratorios con el empeoramiento de sus condiciones de vida y las necesidades vinculadas al ascensor social de la población autóctona entre la cual crece el discurso de la competencia. Como consecuencia, se registran cambios en las estrategias y los proyectos migratorios 
de las personas inmigrantes (retorno o re-emigración), así como el crecimiento de los que viven en la pobreza o con riesgo de exclusión social.

A pesar del contexto de crisis y el mayor rechazo de la población autóctona sin llegar a superar un racismo de baja intensidad, el discurso de los grupos de extranjeros no es muy pesimista según Cea d'Ancona et al. quienes afirman que: "En España vislumbran un futuro incierto, de trabajo y sacrificio, pero a la vez piensan que podrán ver cumplidos algunos sueños que les impelieron a emigrar" (2014:26). Aunque cabe pensar que dicho discurso lo mantienen las personas inmigrantes que permanecen en España a pesar de la crisis, probablemente ocupadas, pero nada tiene que ver con la realidad de las personas que durante estos años han emprendido el viaje de vuelta a su país de origen o han re-emigrado. Así pues, las evidencias analizadas permiten plantear que la integración conseguida durante los años de bonanza económica fue una integración con pies de barro. Es decir, sostenida con falsos valores políticos donde un modelo migratorio esencialmente laboral se presentaba como de integración sociocultural. Con el cambio de ciclo económico y migratorio emerge un nuevo escenario dónde es preciso preguntarse si es posible seguir sin un modelo de integración que defina una estrategia clara y coherente más allá de las necesidades del mercado de trabajo y su gestión. El principal reto pasa por aceptar que no es compatible la integración sociocultural con la gestión de la inmigración únicamente como mano de obra. Es urgente afrontar dicho reto antes que la convivencia muestre más síntomas de debilidad.

\section{Bibliografía}

Alguacil Gómez, J. (2012): "La quiebra del incompleto sistema de Servicios Sociales en España", Cuadernos de Trabajo Social, 25(1), pp. 63-74.

Antón, A. (2013): Ciudadanía activa. Opciones sociopolíticas frente a la crisis sistémica, Madrid, Sequitur.

Aysa-Lastra, M. y L. Cachón (2013): "Movilidad ocupacional segmentada: el caso de los inmigrantes no comunitarios en España", Revista Española de Investigaciones Sociológicas, 144, pp. 23-47.

Borjas, George J. (2003): "The Labor Demand Curve is Downward Sloping: Reexamining the Impact of Immigration on the Labor Market", Quarterly Journal of Economics, 118(4), pp. 1335-1374.

Bourdieu, P. (1994) : Raisons pratique. Sur la théorie de l'action, Paris, du Seuil.

Cachón, L. y M.S. Valles (2003) : "Trade unionism and immigration: reinterprting old and new dilemmas", Transfer, 9(3), pp. 469-482.

Cachón Rodríguez, L. (2009): La "España inmigrante": marco discriminatorio, mercado de trabajo y políticas de inmigración, Barcelona, Anthropos.

Cachón Rodríguez, L. (2012): "Inmigrantes y mercado de trabajo en España en la fase madura del ciclo migratorio", Cuadernos del mercado de trabajo, 8, pp. 42-51.

Cáritas (2013): VIII Informe del Observatorio de la Realidad Social. Empobrecimiento $y$ desigualdad social. Disponible en:

http://www.caritas.es/publicaciones_download.aspx?Id=4706, [consulta 13 de diciembre de 2014]. 
Carrasco, C. y C. García (2012): Inmigración y mercado de trabajo. Informe 2011, Madrid, Ministerio de Empleo y Seguridad Social, Observatorio Permanente de la Inmigración.

Carrasco, R. y C. Ortega (2005): La inmigración en España Características y efectos sobre la situación laboral de los trabajadores nativos, Madrid, Fundación Alternativas.

Carrasco, C. y C. García-Serrano (2015): "Efectos de la crisis en la estructura ocupacional y la biografía de la población inmigrante". Migraciones, 37, pp.75-96.

Cea D'Ancona, Ma Á. y M.S. Valles Martínez (2011): Evolución del racismo y la xenofobia en España Informe 2011, Madrid, OBERAXE, Ministerio de Trabajo e Inmigración.

Cea D’Ancona, Ma Á.; Valles Martínez, M.S y M. Eseverri (2014): “Convergencias y divergencias de los discursos e imágenes de la inmigración en etapas de bonanza y de crisis", Migraciones 35, pp. 11-41.

Cebolla Boado, H. (2010): "Impacto de la inmigración y adecuación de los servicios públicos. La educación”. En: E. Aja; J. Arango y J. Oliver (eds), La inmigración en tiempos de crisis. Anuario de la inmigración en España (edición 2009), Barcelona, CIDOB-Bellaterra.

Cebolla Boado, H. y A. González Ferrer (coords.) (2013): Inmigración ¿integración sin modelo?, Madrid, Alianza.

Colectivo Ioé (2006): Inmigración y vivienda en España, Madrid, Ministerio de Trabajo y Asuntos Sociales, Observatorio Permanente de la Inmigración.

Colectivo Ioé (2012): Impactos de la crisis sobre la población inmigrante. Disponible en: http://www.colectivoioe.org/uploads/0bae582aa3b0842a9eaf50cde16f4f97d9527bcb. pdf [consulta 2 de noviembre de 2014].

Colectivo Ioé (2013): "La población inmigrada ante la crisis: ¿mirando hacia otro lado?", Boletín Ecos, 24, pp. 1-10.

Consejo Económico y Social (2004): La inmigración y el mercado de trabajo en España, Madrid, Consejo Económico y Social, colección informes.

Cuadrado Roura, J.R.; Iglesias Fernández, C. y R. Llorente Heras (2007): Inmigración y mercado de trabajo en España (1995-2005), Bilbao, Fundación BBVA.

Diaz-Serrano, L. (2013): "Immigrants, natives and job quality: evidence from Spain", International Journal of Manpower, 34(7), pp. 753-775.

Domingo, A.; Sabater, A.; Bedoya, M. H y X. Franch (2012): "Regularizaciones y trayectorias de inmigrantes no comunitarios en la provincia de Barcelona", Revista Española de Investigaciones Sociológicas, 140, pp. 121-146.

Fernández, C. y C. Ortega (2008): "Labor market assimilation of immigrants in Spain: Employment at the expense of bad job-matches?", Spanish Economic Review, 10, pp. 83-107. 
Garcés Mascareñas, B.; Franco-Guillén, N. y E. Sánchez-Montijano (2012): “Entre la exclusión y la inclusión. Los discursos políticos en las elecciones municipales de 2011 en Catalunya". En: E. Aja; J. Arango y J. Oliver (eds.), La inmigración en tiempos de crisis. Anuario de la inmigración en España (edición 2009), Barcelona, CIDOB-Bellaterra.

Garrido Medina, L; Miyar Busto, M. y J. Muñoz Comet (2010): "La dinámica laboral de los inmigrantes en el cambio de fase del ciclo económico", Presupuesto y Gasto Público, 61, pp. 201-221.

González Ferrer, A. (2009): "La reagrupación familiar en España. Algunas cifras para el debate". En: E. Aja; J. Arango y J. Oliver (eds.), La inmigración en tiempos de crisis. Anuario de la inmigración en España (edición 2009), Barcelona, CIDOBBellaterra.

Instituto Nacional de Estadística (2009): Encuesta Nacional de Inmigrantes 2007: una monografia, Madrid, INE.

Izquierdo, A. (1996): La Inmigración Inesperada: la población extranjera en España (1991-1995), Madrid, Trotta.

Izquierdo Escribano, A. (2000): "El proyecto migratorio de los indocumentados según género". Papers, revista de sociología, 60, pp. 225-240.

Jódar, P.; Alós, R.; Boso, A.; Guiu, J. y D. Garrell (2014): "La afiliación sindical de la población inmigrante. El caso de CCOO de Cataluña", Cuadernos de Relaciones Laborales, 32(1), pp. 135-163.

Leal, J. y A. Alguacil (2012): "Vivienda e inmigración: las condiciones y el comportamiento residencial de los inmigrantes en España". En: E. Aja; J. Arango y J. Oliver (eds.), La inmigración en tiempos de crisis. Anuario de la inmigración en España (edición 2009), Barcelona, CIDOB-Bellaterra.

Martín Patino, J. M. (coord.) (2011): "Inmigración y crisis económica: el verdadero examen de la integración”. En: VVAA, Informe España 2011, Madrid, Fundación Encuentro.

Martínez de Lizarronda, A. (2009): "La integración de inmigrantes en España: el modelo patchwork", Migraciones, 26, pp.115-146.

Méndez, M.; Cebolla, H. y G. Pinyol (2013): ¿Han cambiado las percepciones sobre la inmigración en España?, Zoom Político, 17, Laboratorio de Alternativas.

Miguélez, F. y P. López Roldán (Coords.) (2014): Crisis, empleo e inmigración en España. Un análisis de las trayectorias laborales, Bellaterra, UAB.

Miguélez, F.; Martín, A.; Alós-Moner, R.; Esteban, F.; López Roldán, P.; Molina, O. y S. Moreno (2011): Trayectorias laborales de los inmigrantes en España, Barcelona, Obra Social de la Caixa.

Ministerio de Trabajo e Inmigración (2009): La muestra continúa de vidas laborales. Descripción general . Disponible en:

http://www.seg-social.es/Internet_1/Estadistica/Est/Muestra_Continua_de_Vidas Laborales/CONTUTIL2009/index.htm, [consulta 2 de noviembre de 2014] 
Morales, L.; Anduiza, E.; Rodríguez, E. y J. San Martín (2008): “Capital social, pautas culturales identitarias y actitudes hacia 'los otros': la incorporación cívica de la población de origen inmigrante en Barcelona y Madrid", Panorama social, 8, pp. 119-142.

Moreno Fuentes, F.J y M. Bruquetas Callejo (2011): Inmigración y estado del bienestar en España, Barcelona, Obra Social de la Caixa.

Muñoz Comet, J. (2011): "Los efectos de la crisis en el empleo de los extranjeros. ¿Importan el nivel educativo y la edad laboral?", Revista Española de Sociología, 16, pp. 9-26.

Muñoz Comet, J. (2014): “¿Qué trabajos ocupan quienes abandonan el desempleo? Diferencias entre españoles y extranjeros en un contexto de cambio económico", Revista Internacional de Sociología, 72(2), pp. 353-376.

OECD (2009): International Migration Outlook 2009, Paris: OECD, SOPEMI.

Pajares, M. (2005): La integración ciudadana. Una perspectiva para la inmigración, Barcelona, Icaria.

Pajares, M. (2007): Inmigración y mercado de trabajo. Informe 2007, Madrid, Ministerio de Trabajo y Asuntos Sociales, Observatorio Permanente de la Inmigración.

Pajares, M. (2010): Inmigración y mercado de trabajo. Informe 2010, Madrid, Ministerio de Trabajo e Inmigración, Observatorio Permanente de la Inmigración.

Parella Rubio, S. (2003): Mujer, inmigrante y trabajadora: la triple discriminación, Barcelona, Anthropos.

Parella, S. y A. Petroff (2014): "Migración de retorno en España: salidas de inmigrantes y programas de retorno en un contexto de crisis". En: J. Arango; D. Moya Malapeira y J. Oliver Alonso (eds.), Inmigración y emigración: mitos y realidades, Anuario de la Inmigración en España (Edición 2014), Barcelona, CIDOB.

Pérez, F. y L. Serrano (2008): "Los inmigrantes y el mercado de trabajo español: una aproximación económica". En: J. García Roca y J. Lacomba (eds.), La inmigración en la sociedad española. Una radiografía multidisciplinar, Barcelona, Bellaterra.

Pérez Infante, J. I. (2008): "El marco legal y la problemática del empleo de los extranjeros en España: una perspectiva económica". En: J. García Roca y J. Lacomba (eds.), La inmigración en la sociedad española. Una radiografia multidisciplinar, Barcelona, Bellaterra.

Pérez Infante, J. I. (2009): “Crecimiento y características del empleo de los inmigrantes en España”, Revista del Ministerio de Trabajo e Inmigración, 80, pp. 237-253.

Portes, A. y R.G. Rumbaut (2010): América inmigrante, Barcelona, Anthropos.

Sarasa, S. y L. Moreno (eds.) (1995): El Estado del Bienestar en la Europa del Sur, Madrid, CSIC.

Solé, C. (1981): La integración Sociocultural de los inmigrantes en Cataluña, Madrid, CIS.

Solé, C. (coord.) (2001): El impacto de la inmigración en la economía y en la sociedad receptora, Barcelona, Anthropos.

Solé, C.; Alcalde, R.; Pont, J.; Lurbe K. y S. Parella (2002): “El concepto de integración desde la sociología de las migraciones", Migraciones 12, pp. 9-41. 
Stanek, M. (2011): "Nichos étnicos y movilidad socio-ocupacional. El caso del colectivo polaco en Madrid", Revista Española de Investigaciones Sociológicas, 135, pp. 69-88.

Subirós, P. (ed.) (2010): Ser immigrant a Catalunya. El testimoni de vint-i-dos protagonistas, Barcelona, Edicions 62.

Viruela, R. (2013): "Entre dos crisis económicas. Estrategias de los rumanos en el mercado de trabajo español”, Política y Sociedad, 50 (3), pp. 981-1008. 